\title{
Modeling of Stress and Strain Distribution in UHMWPE Elements of Knee and Hip Human Joints
}

\author{
M. NABRDALIK* AND M. SOBOCIŃSKI \\ Czestochowa University of Technology, 42-201 Czestochowa, Poland \\ Doi: $10.12693 /$ APhysPolA.138.224 \\ *e-mail: marcin@iop.pcz.pl
}

\begin{abstract}
The paper presents the numerical analysis of stress and strain occurring in the most wearable parts of hip and knee joints endoprostheses. The complexity of the processes taking place in both, natural and artificial joints, makes it necessary to conduct the analysis on the 3D model based on already existing mathematical models. Most of the mechanical failures in alloplasty are caused by material fatigue. To cut down the risk of it, we can either increase the fatigue resistance of the material or decrease the load strain. It is extremely important to indicate the areas where damage or premature wear may occur. The Finite Elements Method makes it possible to calculate the stress and strain in particular elements of the tested models. All presented numerical calculations define quality conclusions concerning the influence of some parameters of endoprostheses on the values of stress and strain that are formed in polyethylene parts of endoprotheses of hip and knee joints. The obtained results help to reveal "weak points" in examined models and thus, counteract the subsequent effects resulting from premature wear of endoprosthesis elements. At the initial study stage, it is appropriate to apply analytic solutions aimed at indicating the areas where damages or premature wear of cooperating elements may occur. The numerical analysis was performed basing on the FEM method using Autodesk Simulation Mechanical 2017 software and the ADINA 7.5.1.
\end{abstract}

topics: endoprothesis, stress, strain, FEM

\section{Introduction}

Fine modelling of physical phenomena is possible only by using complex mathematical description, which in fact, can only be conducted with precise numerical means.

Elaborated algorithms and computer applications in the system significantly improve the endoprosthesis designing process by taking advantages of possibilities given by introduced numerical methods of computing stress and strain in the analyzed model.

\section{Stress and strain analysis for "metal sled-polyethylene insert" in knee endoprosthesis}

The paper's objective was to analyze the state of stress and strain, occurring on the contact surfaces of metal sleds cooperating with polyethylene insert in knee joint endoprosthesis. This is to select the most suitable material which sleds should be made of, in order to achieve an optimum stress distribution on the insert's surface.

The materials for implants should be of low Young's modulus (as close to the bone elasticity as possible), and low weight density. If Young's modulus of the material is too high, like in the case of CoCrMo alloy, it causes decrease of the bone load or its total decay. In such case the implant gets loose because growths and restructuring the bone may only be conducted in proper load conditions. In some cases the titanium alloy is used as well, due to its high mechanical features. Low value of Young's modulus in titanium alloys, may cause decrease of the value of stress generated in polyethylene inserts, and assure more stable implementation of the endoprosthesis in the bone.

It is extremely difficult to find a proper material to build a knee joint endoprosthesis under all those restrictions. One can create a map of stress and strain distribution for each alternative applying FEM in calculations. The calculations are conducted for endoprosthesis with polyethylene insert, and allow to assign reduced and contact stresses occurring in polyethylene inserts cooperating with metal sleds. Figure 1 presents the knee joint endoprosthesis with polyethylene insert. Polyethylene is the weakest point of the endoprosthesis, that is why it is important to present the reduced stress distribution in the inserts.

The following formulas have been adopted for the analysis of the results:

$$
\begin{aligned}
& \sigma_{x}=\frac{E}{1+\nu}\left[\varepsilon_{x}+\frac{\nu\left(\varepsilon_{x}+\varepsilon_{y}+\varepsilon_{z}\right)}{1-2 \nu}\right] \tau_{x y}=G \gamma_{x y} \\
& \sigma_{y}=\frac{E}{1+\nu}\left[\varepsilon_{y}+\frac{\nu\left(\varepsilon_{x}+\varepsilon_{y}+\varepsilon_{z}\right)}{1-2 \nu}\right] \tau_{y z}=G \gamma_{y z} \\
& \sigma_{z}=\frac{E}{1+\nu}\left[\varepsilon_{z}+\frac{\nu\left(\varepsilon_{x}+\varepsilon_{y}+\varepsilon_{z}\right)}{1-2 \nu}\right] \tau_{z x}=G \gamma_{z x}
\end{aligned}
$$




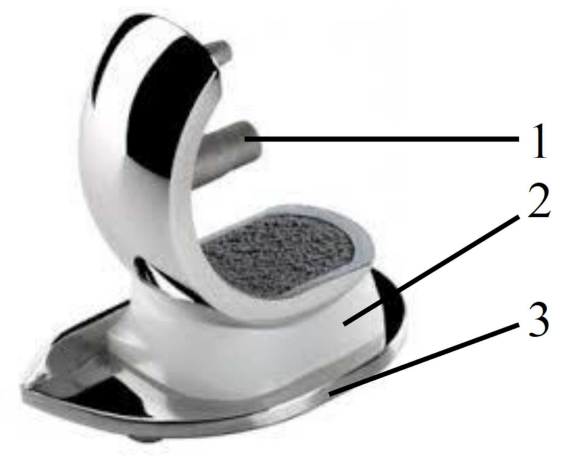

Fig. 1. Knee joint endoprosthesis, 1 - metal sled, 2 - polyethylene insert, 3 - tibia part [1].

These physical equations connect stress and strain tensors' values in 3D, in isotropic, linear-elastic material constans [2-6], i.e., Young's modulus $E$ (elasticity), Kirchoff's modulus $G$ (non-dilatational strain), $\nu$ Poisson's coefficient. When solving (1), we can define components of the stress as a function of the strain.

\subsection{Reduced stress for polyethylene inserts of knee joint}

The durability of endoprosthesis depends on mechanical features of its weakest element, which is PE insert. Reduces stresses are usually normal substitute stress, compared to material strength during one-axis extension. The reduced stresses depend on all stress tensor's components. They cab be represented [2] by expression

or by

$$
\sigma_{\text {red }}=f\left(\sigma_{x x}, \sigma_{y y}, \sigma_{z z}, \tau_{x y}, \tau_{x z}, \tau_{z y}\right) \text {, }
$$

$$
\sigma_{\text {red }}=f\left(\sigma_{11}, \sigma_{22}, \sigma_{33}\right),
$$

where $\sigma_{11}, \sigma_{22}, \sigma_{33}$ denotes the main stress, and $\sigma_{\text {red }}$ is the Huber-Mises's reduced stress.

The Huber-Mises's strength hypothesis can be written [2] as

$$
\begin{aligned}
& \sigma_{\text {red }}=\left[\left(\sigma_{11}-\sigma_{22}\right)^{2}+\left(\sigma_{33}-\sigma_{22}\right)^{2}\right. \\
& \left.+\left(\sigma_{11}-\sigma_{33}\right)^{2}\right]^{1 / 2} / \sqrt{2} .
\end{aligned}
$$

while the stress can be expressed [2] as

$$
\sigma_{i j}=C_{i j k l} \varepsilon_{k l} \text {. }
$$

Each $\delta_{i l}, \delta_{j k}, \delta_{i k}, \delta_{j l}$ is the Kronecker delta, and $C_{i j k l}$ is the resilience tensor, defined as [2]:

$$
\begin{aligned}
& C_{i j k l}=\frac{E}{2(1+\nu)} \\
& \quad \times\left(\delta_{i l} \delta_{j k}+\delta_{i k} \delta_{j l}+\frac{2 \nu}{1-2 \nu} \delta_{i j} \delta_{k l}\right) .
\end{aligned}
$$

\subsection{Criteria included in calculations}

The contact stress occurs when two elements press to each other with a force. This takes place in a certain area and can reach quite high values

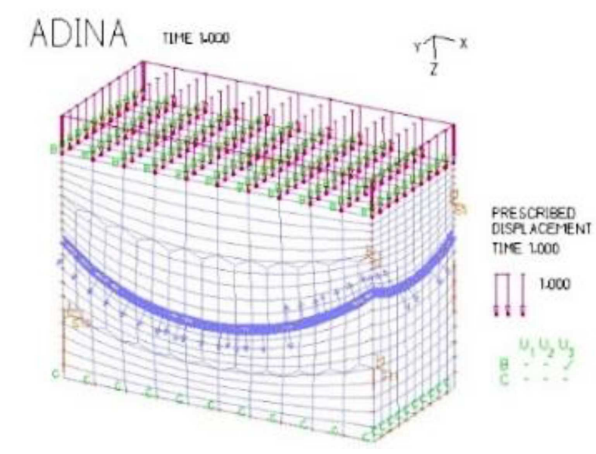

Fig. 2. Numerical model used for calculations.

even at respectively low value of clamp. Theoretical criteria for contact stress are applied according to Hertz's theory. The following conditions occur:

- contacting elements are made of homogeneous isotropic materials and Hooke's law wise,

- the surfaces are fixed in the contact area of the element with smooth and regularly curved surfaces,

- when subjected to load there are only slight strains in the contact area,

- the contact area is relatively small when compared with the surfaces of the contacting elements,

- on the contact area there only occur normal strains.

Numerical model is a simplified version of the original endoprosthesis, though the sleds' geometry has been maintained. Figure 2 presents the numerical model.

\subsection{Results analysis with Finite Elements Method and ADINA SYSTEM 8.6}

The calculations prove that stress in endoprosthesis is concentrated in the polyethylene insert, directly below the contact area of both elements. The highest stress is located right underneath the insert's surface. Point in which stresses are characterized by the biggest value, is called Bielajew's point. Some examples of the calculations are shown in Figs. 3 and 4.

\section{Numerical analysis in elements of hip joint endoprosthesis}

Cooperation between the system elements head - cup is particularly important due to a complex type of strain and stress that occurs in an artificial hip joint. These cooperation processes are relevant also for other parts and elements of the endoprosthesis and tissues surrounding the implant. At the initial stage of the endoprosthesis design, one should use analytical solutions aimed to indicate the areas where damages or premature wear of 


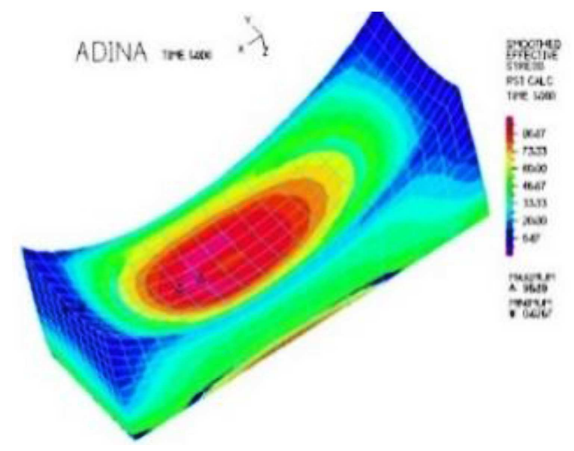

Fig. 3. Contact stress distribution occurring in a spherical polyethylene insert. Spherical insert $8 \mathrm{~mm}$ thick cooperates with a sled of radius $17 \mathrm{~mm}$. Load $1500 \mathrm{~N}$.

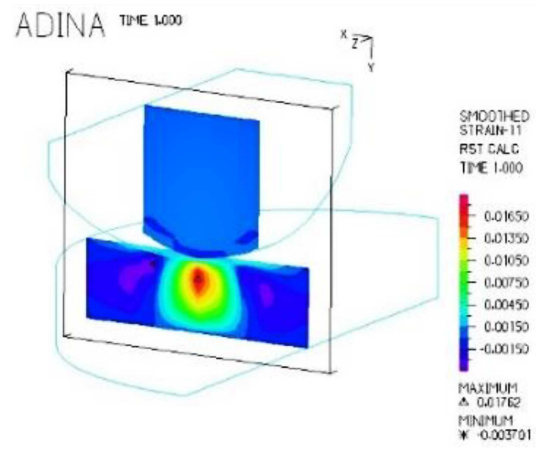

Fig. 4. Strain distribution occurring in spherical polyethylene insert. Spherical insert $8 \mathrm{~mm}$ thick, cooperates with sled of radius $27 \mathrm{~mm}$. Load $1500 \mathrm{~N}$.

the components are most likely to occur [1, 7, 8]. Most of mechanical damages in hip joint replacement are due to material fatigue. All the presented numerical computations allow to draw valuable conclusions concerning the effect of selected functional parameters on the level of stresses and strains in individual components of the endoprosthesis [9].

\subsection{Analysis of stress and strain distribution}

The model of endoprosthesis is composed of the stem fixed by means of a lock screw titanium or ceramic head, acetabulum and the acetabulum housing [10]. Simulations were conducted on pairs:

1. endoprosthesis ceramic head - acetabulum UHMWPE,

2. endoprosthesis metal head - acetabulum UHMWPE.

Numerical analysis was performed using the FEM by means of Autodesk Simulation Mechanical 2017 software.

The model was restrained at all external surfaces of the stem, and it simulated stabilization in the bone tissue. Figure 5 illustrates the surfaces to which the loads with the values of $600 \mathrm{~N}$ and $750 \mathrm{~N}$ were applied.

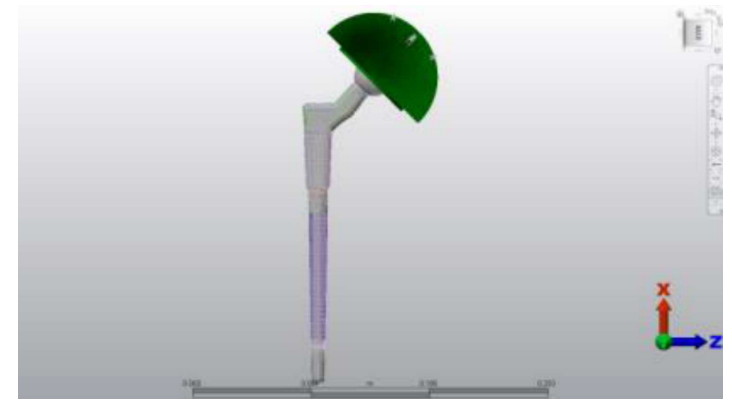

Fig. 5. Surface where the loads were applied.

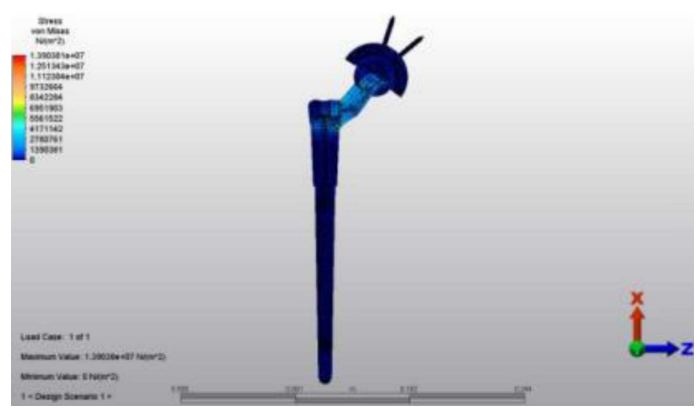

Fig. 6. Distribution of stress in the analyzed system at the load of $750 \mathrm{~N}$. Endoprosthesis head made of $\mathrm{ZrO}_{2}$ ceramics.

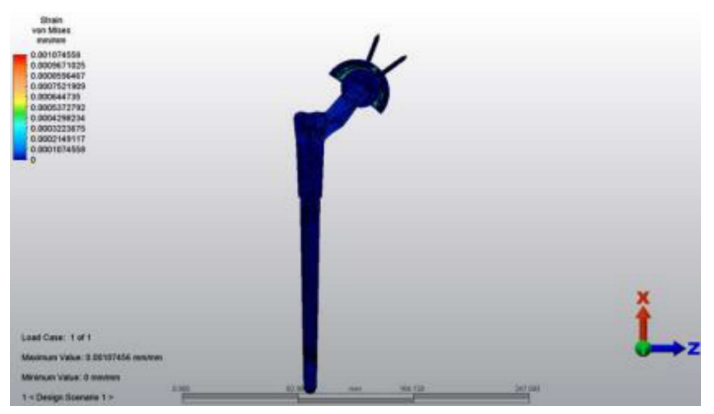

Fig. 7. Distribution of strain in the analyzed system at the load of $600 \mathrm{~N}$. Endoprosthesis head made of $\mathrm{Ti}_{6} \mathrm{Al}_{4} \mathrm{~V}$ alloy.

Figure 6 illustrates examples of the stress distributions of the ceramic head and UHMWPE acetabulum. In turn, Fig. 7 illustrates examples of the strain distributions (cross-section of the analyzed system) for the pair of the metal head and UHMWPE acetabulum. The highest values of stress at the load of $600 \mathrm{~N}$ and $750 \mathrm{~N}$ were observed in the endoprosthesis head just at the stem collar. The obtained values are $11 \mathrm{MPa}$ for the loading force of $600 \mathrm{~N}$, and $14 \mathrm{MPa}$ for the force of $750 \mathrm{~N}$.

As expected, maximal stresses were observed in the acetabulum made of UHMWPE. However, the levels they reached are very low. It can be concluded that they do not have an effect on the system stability. 


\section{Conclusions}

1. An important element influencing durability of endoprostheses is optimizing of the geometry of the implants in the friction node.

2. The analysis performed in the study revealed that the values of stresses and strains in the analysed system at the adopted load similar to that in natural conditions are low. They do not cause formation of locations with accumulated stresses.

3. Polyethylene components are not exposed to substantial strains, which can be a good predictor of a longer life of these components. The findings of the study lead to the conclusion that the adopted geometrical solutions are optimal and allow for long and failure-free use.

\section{References}

[1] Ch.F. Scifert, T. Brown, J. Lipman, Clin. Biomech. 14, 697 (1999).

[2] O.C. Zienkiewicz, FEM, Publishing Arkady, 1972.
[3] M. Gierzyńska-Dolna, Biotribology Publishing of Czestochowa University of Technology, 2002.

[4] M. Olinski, A. Gronowicz, A. Handke, M. Ceccarelli, Int. J. Appl. Mech. Eng. 21, 611 (2016).

[5] J. Marciniak, Biomaterials, Publishing of Silesian University of Technology, Gliwice 2002.

[6] M. Krzywicka, J. Grudziński, J. Tatarczak, Inżynieria Materiałowa 37, 97972 (2016) (in Polish).

[7] A. Polyakov, V, Pakhaliuk, M. Kalinin, V. Kramar, Proc. Eng. 100, 530 (2015).

[8] F. El-Shiekh, E.D. Hussam, Ph.D. Thesis, Dublin City University 2002.

[9] A. Jahnkea, C. Ulloaa, J. Seegera, Clinical Biomechanics 52, 49 (2018).

[10] Zimmer Biomet. 